\title{
Erratum to: Multivariate Epi-splines and Evolving Function Identification Problems
}

\author{
Johannes O. Royset $^{1} \cdot$ Roger J-B Wets ${ }^{2}$
}

Received: 6 April 2016 / Accepted: 17 October 2016 / Published online: 2 November 2016

(C) Springer Science+Business Media Dordrecht 2016

\section{Erratum to: Set-Valued Var. Anal DOI 10.1007/s11228-015-0329-4}

The definition of infinite refinement in Royset \& Wets (2016) needs to be strengthened to exclude refinements, as illustrated by an example, that would not become fine enough. Without this strengthening, some results in that paper will not hold.

The definition of an infinite refinement needs to be adjusted as follows:

Definition 3.4 (infinite refinement) A sequence $\left\{\mathcal{R}^{\nu}\right\}_{\nu=1}^{\infty}$ of partitions of a closed set $B \subseteq$ $\mathbb{R}^{n}$, with $\mathcal{R}^{v}=\left\{R_{k}^{v}\right\}_{k=1}^{N^{v}}$, is an infinite refinement if

$$
\begin{aligned}
& \text { for every } x \in B \text { and } \varepsilon>0, \text { there exist } \bar{v} \in \mathbb{N} \text { and } \delta \in(0, \varepsilon) \text { such that } \\
& R_{k}^{v} \subset \mathbb{B}(x, \varepsilon) \text { for every } v \geq \bar{v} \text { and } k \text { satisfying } R_{k}^{v} \cap \mathbb{B}(x, \delta) \neq \emptyset .
\end{aligned}
$$

The reason is that the corresponding definition in [1] allows for the possibility to choose a somewhat pathological refinement that would fail to become sufficiently "small" as illustrated next:

The online versions of the original articles can be found at http://dx.doi.org/10.1007/s11228-015-0329-4.

Johannes O. Royset

joroyset@nps.edu

Roger J-B Wets

rjbwets@ucdavis.edu

1 Operations Research Department, Naval Postgraduate School, Monterey, CA, USA

2 Department of Mathematics, University of California, Davis, CA, USA 
Example Let $B=[0, \infty) \subset \mathbb{R}$ and the infinite refinement be partitioning $(0, \sqrt{v})$ mostly into segments of length $1 / \sqrt{v}$, with the exception that a portion of the segment nearest the origin is added to the final segment covering $(\sqrt{v}, \infty)$. Specifically, we let $N^{v}=v+1$, $R_{1}^{v}=(0,1 /(4 \sqrt{v})) \cup(1 /(2 \sqrt{v}), 1 / \sqrt{v}), R_{k}^{v}=((k-1) / \sqrt{v}, k / \sqrt{v})$ for $k=2, \ldots, N^{v}-1$, and $R_{N^{v}}^{v}=(\sqrt{v}, \infty) \cup(1 /(4 \sqrt{v}), 1 /(2 \sqrt{v}))$. Clearly, for every $v \in \mathbb{N},\left\{R_{k}^{v}\right\}_{k=1}^{N^{v}}$ is a partition of $B$. Also, $\left\{\mathcal{R}^{\nu}\right\}_{\nu=1}^{\infty}$ would be an appropriate infinite refinement of $B$ according to [1], which requires only:

$$
\begin{aligned}
& \text { for every } x \in B \text { and } \varepsilon>0, \text { there exists } \bar{v} \in \mathbb{I} \text { such that } \\
& R_{k}^{v} \subset \mathbb{B}(x, \varepsilon) \text { for every } v \geq \bar{v} \text { and } k \text { satisfying } x \in \operatorname{cl} R_{k}^{v} .
\end{aligned}
$$

We establish that this requirement is satisfied: Let $x \in B$ and $\varepsilon>0$ be arbitrary. First, suppose that $x=0$. Then, set $\bar{v} \geq 1 / \varepsilon^{2}$. In this case, we have that $x \in \operatorname{cl} R_{1}^{v}$ for all $v$. Since $R_{1}^{v} \subset[0,1 / \sqrt{v}] \subset[0, \varepsilon]$ for all $v \geq \bar{v}$, the requirement $R_{1}^{v} \subset \mathbb{B}(x, \varepsilon)$ is satisfied. Second, suppose that $x>0$. Then, set $\bar{v}>\max \left\{4 / \varepsilon^{2}, x^{2}, 1 /\left(4 x^{2}\right), 1\right\}$. In this case, $1 /(2 \sqrt{v})<x<\sqrt{v}$ for $v \geq \bar{v}$. Consequently, $x \notin \operatorname{cl} R_{N^{v}}^{v}$ for any $v \geq \bar{v}$. We therefore have that for each $v \geq \bar{v}$, there exists a $k^{\nu}(x) \in\left\{1,2, \ldots, N^{v}-2\right\}$ such that $x \in \operatorname{cl} R_{k^{v}(x)}^{v} \cup \operatorname{cl} R_{k^{v}(x)+1}^{v}$ and $x \notin \mathrm{cl} R_{k}^{v}$ for all $k \in\left\{1,2, \ldots, N^{v}\right\} \backslash\left\{k^{v}(x), k^{v}(x)+1\right\}$. Since for each $v \geq \bar{v}, R_{k^{v}(x)}^{v} \cup R_{k^{v}(x)+1}^{v}$ is contained in a line segment of length at most $\varepsilon$ the requirement holds. We have shown that $\left\{\mathcal{R}^{\nu}\right\}_{\nu=1}^{\infty}$ satisfies the condition of the original definition in [1]. This (pathological) infinite refinement makes the proofs of Theorems 3.5, 3.8, and 3.11 in [1] invalid due to the fact that there are points in $R_{N^{v}}^{v}$ that are arbitrarily closed to 0 , but $R_{N^{\nu}}^{v}$ is not becoming arbitrarily "small." The example fails the condition of the adjusted definition given here as there is no $\delta \in(0, \varepsilon)$ such that $R_{N^{v}}^{v} \cap \mathbb{B}(0, \delta)=\emptyset$ for all $v$. We observe that all "natural" partitions such as the one with $R_{k}^{v}=((k-1) / \sqrt{v}, k / \sqrt{v})$ for $k=1, \ldots, N^{v}-1$, and $R_{N^{v}}^{v}=(\sqrt{v}, \infty)$ satisfy the condition of the adjusted definition.

With the adjusted definition of infinite refinement, Theorems 3.5, 3.8 and 3.11 in [1] are correct as stated. However, we provide below an updated version of the proof of Theorem 3.5 that makes the necessary adjustments; some typos are also corrected and we provide some additional explanation. Corrected proofs of Theorems 3.8 and 3.11 follow the same pattern.

Theorem 3.5 (dense approximation) For any $p \in \mathbb{N _ { 0 }}$ and $\left\{\mathcal{R}^{v}\right\}_{\nu=1}^{\infty}$, an infinite refinement of a closed set $B \subseteq \mathbb{R}^{n}$,

$$
\bigcup_{\nu=1}^{\infty} \mathrm{e}-\operatorname{spl}_{n}^{p}\left(\mathcal{R}^{\nu}\right) \text { is dense in lsc-fcns }(B) \text {. }
$$

Proof Let $s^{0} \in \operatorname{lsc}$-fens $(B)$ and $\mathcal{R}^{v}=\left\{R_{k}^{v}\right\}_{k=1}^{N^{v}}$. It suffices to construct a sequence of epi-splines of order $p=0$. For every $v \in \mathbb{N}$ and $R_{k}^{v}, k=1,2, \ldots, N^{v}$, we define

$$
\sigma^{v}\left(R_{k}^{v}\right):= \begin{cases}\inf _{x \in \operatorname{cl} R_{k}^{v}} s^{0}(x) & \text { if } \inf _{x \in \operatorname{cl} R_{k}^{v}} s^{0}(x) \in[-v, v] \\ v & \text { if } \inf _{x \in \operatorname{cl} R_{k}^{v}} s^{0}(x)>v, \\ -v & \text { otherwise }\end{cases}
$$

and construct $s^{v}: \mathbb{R}^{n} \rightarrow \overline{\mathbb{R}}$ as follows:

$$
s^{v}(x):=\min _{k=1,2, \ldots, N^{v}}\left\{\sigma^{v}\left(R_{k}^{v}\right) \mid x \in \mathrm{cl} R_{k}^{v}\right\}, x \in B,
$$


and $s^{\nu}(x)=\infty$ for $x \notin B$. Clearly, $s^{\nu}$ is constant on each $R_{k}^{v}, k=1,2, \ldots, N^{\nu}$ and $\liminf _{x^{\prime} \rightarrow x} s^{\nu}\left(x^{\prime}\right)=s^{\nu}(x)$ for all $x \in \mathbb{R}^{n}$. Hence, $s^{\nu} \in \mathrm{e}-\operatorname{spl}_{n}^{0}\left(\mathcal{R}^{v}\right)$ and consequently also in e-spl ${ }_{n}^{p}\left(\mathcal{R}^{v}\right)$ for $p \in \mathbb{N}$. We next show that the two conditions of Proposition 2.1 in [1] hold. Let $x \in B$ and $\varepsilon>0$ be arbitrary. By lower semicontinuity of $s^{0}$ there exists $\delta>0$ such that $s^{0}(z) \geq s^{0}(x)-\varepsilon$ whenever $z \in \mathbb{B}(x, \delta)$. Since $\left\{\mathcal{R}^{\nu}\right\}_{\nu=1}^{\infty}$ is an infinite refinement, there also exist $\bar{v}$ and $\gamma \in(0, \delta)$ such that $R_{k}^{v} \subset \mathbb{B}(x, \delta)$ for every $v \geq \bar{v}$ and $k$ satisfying $R_{k}^{v} \cap \mathbb{B}(x, \gamma) \neq \emptyset$. Hence, for $y \in \mathbb{B}(x, \gamma / 2) \cap B, s^{v}(y) \geq \min _{k}\left\{\min \left\{v, \inf _{z \in \operatorname{cl} R_{k}^{v}} s^{0}(z)\right\}\right.$ : $\left.y \in \operatorname{cl} R_{k}^{v}\right\} \geq \min \left\{v, s^{0}(x)-\varepsilon\right\}$ for all $v \geq \bar{v}$. Since $s^{\nu}(y)=\infty$ for $y \notin B$, we thus have that for every sequence $x^{v} \rightarrow x$,

$$
\liminf _{v} s^{v}\left(x^{v}\right) \geq \liminf _{v} \min \left\{v, s^{0}(x)-\varepsilon\right\}=s^{0}(x)-\varepsilon .
$$

Since $\varepsilon$ is arbitrary, $\liminf s^{\nu}\left(x^{\nu}\right) \geq s^{0}(x)$. Because $B$ is closed, this inequality also holds for $x \notin B$. Thus, condition a) of Proposition 2.1 in [1] is established. For b), simply set $x^{v}=$ $x$ for all $v$. If $x \notin B$, then $s^{\nu}\left(x^{\nu}\right)=s^{0}(x)=\infty$. If $x \in B$, then $s^{\nu}(x) \leq \max \left\{-v, s^{0}(x)\right\}$. From this follows that for $x \in \mathbb{R}^{n}$,

$$
\limsup _{v} s^{v}\left(x^{v}\right)=\limsup _{v} s^{v}(x) \leq s^{0}(x),
$$

which establishes part $b$ ) of [1, Proposition 2.1] and concludes the proof.

It is the existence of a neighborhood of $x$ for which all points $y$ satisfy

$$
\min _{k}\left\{\min \left\{v, \inf _{z \in \mathrm{cl} R_{k}^{v}} s^{0}(z)\right\}: y \in \operatorname{cl} R_{k}^{v}\right\} \geq \min \left\{v, s^{0}(x)-\varepsilon\right\}
$$

that might fail in the original proof resulting in the possibility that the following inequality in the proof would not hold.

\section{References}

1. Royset, J.O., Wets, R.J.-B.: Multivariate epi-splines and evolving function identification problems. SetValued Var. Anal., to appear (2016) 